(Aus dem gerichtl.-med. Institut der Universität Genua [Prof. A. Severi].)

\title{
Zur Kenntniss \\ der Duodenalverdauung des Eiweisses, in der Leiche untersucht.
}

Von

Dr. Carlo Ferrai, Assistent.

(Mit 3 Textfiguren.)

Als ich voriges Jahr das Ergebniss meiner Untersuchungen über die postmortale Verdauung des Eiweisses im Magen veröffentlichte, versprach ich auch die von mir gleichzeitig über die duodenale Verdauung unter gleichen Bedingungen angestellten Versuche nach ihrer Vervollständigung mitzutheilen. Eigentlich berührt die Ergründung dieses Problems nicht direct die gerichtlich-medicinische Praxis, immerhin scheint mir dasselbe von ungemeinem tanatologischem Interesse $\mathrm{zu}$ sein, indem es uns Gelegenheit bietet, das Vorkommen und Fortbestehen gewisser Vorgänge in der Leiche näher zu erforschen, die bedingt sind entweder durch eine überlebende Parenchymalthätigkeit oder durch die bereits im Leben gebildeten Secretionsproducte derselben. Andererseits bot sich mir dadureh die erwünschte Gelegenheit, gewisse Einzelheiten der Eiweissverdauung im Duodenum nach einer neven Methode zu erforschen, die vielleicht dazu geeignet sein dürfte, einige neue Thatsachen zu der reichen Fülle von hochwichtigen Ergebnissen, die Paulow und seine Schüler der Wissenschaft errangen, hinzuzufügen.

Zuerst wandte ich mich dem Studium der proteolitischen Wirkung des Duodenuminhalts zu, als diejenige, die am leichtesten messbar und in Zahlen auszudrücken ist. Als Versuchsobject diente mir geronnenes Eiweiss, das ich dadurch erhielt, dass ich ganz frische Eier während $10 \mathrm{Min}$. in siedendem Wasser kochen liess und dann aus denselben kleine Kuben von genau $250 \mathrm{mg}$ Gewicht herausschnitt. Selbstverständlich wurden diese Würfelchen jedes Mal 
vor dem Versuche, in dem sie Verwendung fanden, frisch präparirt, und damit sie ja nichts durch Verdunstung an Gewicht verlieren, wurden sie für die Dauer der wenigen Augenblicke, die zwischen ihrer jedesmaligen Herstellung und Verwendung verflossen, in einer feuchten Kammer aufbewahrt.

War die Zeit da, zur Operation vorzuschreiten, wurde der Hund schnell gefesselt und festgelegt: die Bauchhöhle wurde unterhalb des Rippenbogens eröffnet, der Magen herausgezogen und mittelst einer, um die untere Magenöffnung locker gelegte Schlinge isolirt. Hierauf wurde die Magenwand aufgeschnitten, die untere Magenöffnung mit einem Wattebausch sorgfältig ausgeputzt, und während ein Assistent die beiden Magenzipfel aus einander hob, um eine Beschmutzung des Operationsfeldes durch den Mageninhalt zu vermeiden, schob ich durch die nunmehr leicht zugängliche Oeffnung des Pilorus rasch acht der bereit gehaltenen Eiweisskuben in den Zwölffingerdarm, zog die Schlinge augenblicklich zu und brachte den Magen in die Baucbhöhle zurück; dann wurden die Ränder der Bauchwand fest zusammengeklemmt und der Hund vermittelst Bulbärstiches getödtet. Die ganze Operation dauerte natürlich wenige Augenblicke, so dass auch in den ungünstigsten Fällen zwischen der Einführung des ersten Würfels in den Darm und dem Bulbärstich nie mehr als ${ }^{3 / 4}$ bis 1 Min. verflossen, dabei unterblieb grundsätzlich die Verwerthung derjenigen Versuche, deren Daner diese Grenzen aus irgend einer Ursache überschritt.

Die Leichen der Thiere wurden auf die Seite gelegt und horizontal gelagert in einem nicht allzu schroffen Temperaturschwankungen ausgesetzten Raum; darauf erfolgte zur bestimmten Zeit die Section, die Klemmen wurden entfernt, die Kuben sorgfältig herausgezogen und gereinigt und ein jedes derselben einzeln gewogen.

Die $z u$ diesen Versuchen verwendeten Hunde waren möglichst von annähernd gleichem Gewicht und immer von geringer Grösse. Es wurde, soweit als möglich, dafür Sorge getragen, dass sich die Thiere immer unter gleichen Experimentalbedingungen befanden, und ganz besonders achtete ich darauf, dass sie seit einiger Zeit an Fleischkost gewöhnt waren; wer Paulow's Untersuchungen kennt, wird diese Vorsichtsmaassregeln vollkommen rechtfertigen. Wirklich hatte ich bei einer ganzen Anzahl Versuche, den ersten, bemerkt, dass die Ergebnisse nicht sehr in Einklang unter einander standen, und jch öfters Zahlen erhielt, die weit von den erwarteten ab- 
wichen; sämmtliche Thiere stammten aus dem städtischen Stabularium, und ich erklärte mir diesen Umstand leicht aus der Verschiedenartigkeit der Kost, an welche die Thiere je nach der ökonomischen Lage und Freundlichkeit ihres ehemaligen Herren gewöhnt waren. Dass ein solcher Umstand thatsächlich in Stande ist, die Versuchsergebnisse merklich zu stören, erhellt aus den Versuchen Wassiljew's. Dieser fand, dass ein während anderthalb Monaten ausschliesslich mit Milchsuppe ernährter Hund, dessen Pankreassaft während der ersten sechs Stunden nach der î̃ahlzeit ein Verdauungsvermögen von kaum $0,25 \mathrm{~mm}$ (nach der Mett'schen Methode gemessen) besass, sobald er einige Tage lang mit Fleisch gefüttert wrurde, in den sechs unmittelbar auf die Mahlzeit folgenden Stunden einen Pankreassaft abzusondern vermochte, dessen proteolitische Wirkung $3,5 \mathrm{~mm}$ gleich kam. Und umgekehrt liess Jablonsky einen an Fleischkost gewöhnten Hund während einer gewissen Zeit nur Milchsuppe verabreichen; die anfangs sehr starke proteolitische Wirkung des Pankreassaftes dieses Thieres war bereits nach 30, 40, 52,76 Tagen auf $4-2,2-1,25-0 \mathrm{~mm}$ (nach Mett) herab gesunken.

Eine erste Versuchsreihe wurde in der Absicht angestellt, den Verlauf der Eiweissverdaung im Dnodenum des Cadavers, je nach der mehr oder minder langen Zeit, während welcher die Eiweisskuben selbst im Darme verblieben, zu ermitteln. Da das Duodenum zu jeder Zeit der Verdauung eine bestimmte Menge Pankreassaft von bestimmter Concentration enthalten muss, ist es leicht verständlich, dass das in dasselbe eingeführte geronnene Eiweiss alsbald der tripsinischen Verdauung anheimfällt, ähnlich wie die Eiweissröhrchen, die Mett in einer Wärmekammer der Einwirkung des Pankreassaftes aussetzte. Bei diesen vollzog sich die Verdauung im Brutofen bei constanter Temperatur und in ganz reinem Pankreassaft, und daher war auch ihre Intensität eine der Dauer direct proportionale, wie bereits Ssamojloff nachgewiesen hatte. Das verschiedene Medium, die Erkaltung der Leiche, die durch die Anhäufung der Verdauungsproducte in einer beschränkten Flüssigkeitsmenge bedingte Abschwächung und Hemmung des Verdauungsvermögens der Enzyme, trugen in unserem Falle dazu bei, ganz verschiedene Verhältnisse zu schaffen, die auch den Verlauf der $\mathbf{E r}$ scheinung wesentlich verschieden gestalten mussten. Eben der Einfluss dieser Bedingungen war es, den ich zunächst in dieser Ver- 
suchsreihe näher zu ermitteln beabsichtigte, indem ich mir die Frage stellte: In welchem Maasse und auf welche Dauer entfaltet der Duodenuminhalt eines in vollem Gange der Verdauung getödteten Thieres seine proteolitische Wirkung? Die zu diesem Zwecke angestellten Versuche waren fünf an der Zahl. Die Einführung der Eiweisskuben und zugleich die Tödtung der Thiere erfolgte jedes Mal anderthalb Stunden nach der Fütterung, die Würfel verblieben 2, 4, 6, 8 und 10 Stunden im Darm des Cadavers. Ein längeres Verbleiben der Eiweisskuben in demselben wurde vermieden, um möglichst das Dazwischentreten von Fäulnissvorgängen zu verhüten, dann aber auch, weil die Verdauungsprocesse inzwischen fast vollkommen erloschen waren.

\section{Versuch.}

Hund O. Gewicht ca. $6 \mathrm{~kg}$. 15. Juni 1900. Aeussere Temperatur $27^{\circ} \mathrm{C}$.

Um $11^{\mathrm{h}} 30^{\prime}$ Nachm. bekommt das Thier etwas gesottenes Fleisch. $3^{\mathrm{h}}$ : Laparotomie, Eröffnung des Magens und Einführung durch den unteren Magenmund in den $Z$ wölffingerdarm von 8 Eiweisswürfeln von je $250 \mathrm{mg}$ Gewicht. Die Schlinge um den Pilorus wird angezogen, die Bauchhöhle festgekiemmt und das Thier vermittelst Bulbärstiches getödtet. $5^{\mathrm{h}}$ Abends: Obduction. Rectaltemperatur $36^{\circ}$. Die Eiweisskuben erscheinen gelb verfärbt; ihre Oberfläche zeigt eine zarte Körnung, und die Würfel selbst lassen sich mit Ausnahme des mittleren Kernes leicht zwischen den Fingern zerreiben.

Gewichtsverlust der Kuben (ursprüngliches Gewicht $250 \mathrm{mg}$ ) nach 2 Stunden postmortaler Verdauung: $80,84,79,68,74,91,77,81 \mathrm{mg}$; mittlerer Verlust $79 \mathrm{mg}$ $=31,5 \%$, pro Stunde $15,8 \%$.

\section{Versuch.}

Hund P., weiblichen Geschlechts, wiegt ungefähr $5 \mathrm{~kg}$. 14. Juli 1900. Aeussere Temperatur $26-28^{\circ} \mathrm{C}$. Nachm. $1^{\text {h }}$ : Verabreichung von etwas Fleisch. 2h $30^{\prime}$ Laparotomie; Einführung von 8 Eiweisskuben in das Duodenum; Tödtung. 6 h $30^{\prime}$ : Obduction. Rectaltemperatur $32,1^{\circ}$. Die Würfel erscheinen stark gelb verfärbt; ihre Korrosion ist nicht viel deutlicher ausgeprägt als bei vorigem Versuch, doch ist ihre äussere Schicht in hohem Maasse zwischen den Fingern zerreibbar.

Gewichtsabnahme nach 4 Stunden postmortaler Verdauung (ursprüngliches Gewicht $250 \mathrm{mg}$ ): 110, 122, 118, 106, 103, 125, 117, $111 \mathrm{mg}$; mittlere Abnahme $114 \mathrm{mg}=45,5 \%$, pro Stunde $11,4 \%$.

\section{Versueh.}

Hund R. Gewicht ca. 5 kg. 21. Juni 1900. Aeussere Temperatur $25-27^{\circ} \mathrm{C}$.

1/28 h Morgens wird dem Thier etwas Fleisch verabreicht. Um 10 $\mathrm{h}$ : Eröffnung des Magens und Einführung von 8 Eiweisskuben von je $250 \mathrm{mg}$ in den Zwölffingerdarm. Nachm. $4^{\mathrm{h}}$ : Obduction. Rectaltemperatur $27^{\circ} \mathrm{C}$. Würfel tiefgelb, breiig, zerreiblich. 
Gewichtsverlust der Kuben während 6 Stunden postmortaler Verdauung (eines derselben konnte nicht nachgewogen werden wegen zufälliger Verbröckelung) $139,132,150,135,112,142,149 \mathrm{mg}$; mittlerer Verlust $137 \mathrm{mg}=54,9 \%$, pro Stunde $9,1 \%$.

\section{Versuch.}

Hund S. Gewicht ca. 6 kg. 27. Juni 1900. Aeussere Temperatur $24-26^{\circ} \mathrm{C}$.

$9 \mathrm{~h} 30^{\prime}$ : Das Thier bekommt etwas gesottenes Fleisch. 11 h : Laparotomie; Einführung durch den Magen in das Duodenum von 8 Kuben geronnenes Eiweiss im Gewicht von je $250 \mathrm{mg}$. Unmittelbar darauf wird das Thier getödtet. $7 \mathrm{~h}$ Abends: Obduction. Rectaltemperatur $26,2^{\circ} \mathrm{C}$. Würfel tief orangegelb verfärbt, stark reducirt und bröcklig.

Gewichtsabnahme der einzelnen Kuben nach 8 Stunden postmortaler Verdauung: 138,149,142,125,157,150,155, $167 \mathrm{mg}$; mittlerer Verlust $147,8 \mathrm{mg}=$ $59,1 \%$, pro Stunde $7,5 \%$.

\section{Versuch.}

Hund T. Geschlecht weiblich. Gewicht ca. $5 \mathrm{~kg}$. 30. Juni 1900. Aeussere Temperatur $25--27^{\circ} \mathrm{C}$.

Um $8^{\mathrm{h}}$ wird dem Thier etwas gesottenes Fleisch dargereicht. $91 / 2 \mathrm{~h}:$ Eröffnung des Magens und Einführung in den Zwölffingerdarm von 8 Kuben geronnenes Eiweiss im Gewicht von je $250 \mathrm{mg}$. Tödtung des Thieres mittelst Bulbärstiches. Abends $7 \mathrm{~h}$ 30': Obduction. Rectaltemperatur $26,6^{\circ}$ C. Die Kuben zeigen das gleiche Aussehen wie oben.

Ihr Gewichtsverlust während 10 Stunden postmortaler Verdauung beträgt: $149,140,152,128,163,151,159,157 \mathrm{mg} ;$ mittlerer Verlust $151 \mathrm{mg}=60,4 \%$, pro Stunde $6 \%$.

Obige Ergebnisse habe ich in folgender Tabelle übersichtlich zusammengefasst.

$$
\text { Tabelle I. }
$$

\begin{tabular}{|c|c|c|c|c|c|c|c|c|}
\hline \multirow[t]{2}{*}{ Hund } & \multirow{2}{*}{$\begin{array}{c}\text { Ge- } \\
\text { wicht } \\
\mathrm{kg}\end{array}$} & \multirow{2}{*}{$\begin{array}{l}\text { Tem- } \\
\text { peratur } \\
0 \mathrm{C} .\end{array}$} & \multirow{2}{*}{$\begin{array}{l}\text { Verbleib } \\
\text { d. Kuben } \\
\text { im Duo- } \\
\text { denum } \\
\text { Stunden }\end{array}$} & \multirow{2}{*}{$\begin{array}{l}\text { Rectal- } \\
\text { tempera- } \\
\text { tur z. Zeit } \\
\text { d. Obduc- } \\
\text { tion 0 C. }\end{array}$} & \multicolumn{2}{|c|}{$\begin{array}{c}\text { Totale mittlere } \\
\text { Gewichtsabnahme } \\
\text { per } 250 \mathrm{mg}\end{array}$} & \multirow{2}{*}{$\begin{array}{c}\text { Mittlere } \\
\text { Gewichts- } \\
\text { abnahme } \\
\text { p. Stunde } \\
\% / 0\end{array}$} & \multirow{2}{*}{$\begin{array}{l}\text { Abnahme } \\
\text { p. Stunde } \\
\text { ber. von } \\
2 \text { zu } 2 \text { St. } \\
0 \%\end{array}$} \\
\hline & & & & & $\mathrm{mg}$ & $\%$ & & \\
\hline o. & 6 & 27 & 2 & 36,0 & 79 & 31,5 & 15,8 & $\begin{array}{c}15,8 \\
\text { 1. u. } 2 \text { St. }\end{array}$ \\
\hline P. & 5 & 28 & 4 & 32,1 & 114 & 45,5 & 11,4 & 3. u. ${ }^{7}$. St. \\
\hline R. & 5 & 26 & 6 & 27,6 & 137 & 55 & 9,1 & $\begin{array}{c}4,7 \\
\text { 5. u. } 6 . \text { St. }\end{array}$ \\
\hline $\mathrm{S}$. & 6 & 25 & 8 & 26,2 & 148 & 59 & 7,5 & 7. u. $\stackrel{2}{8 . \text { St. }}$ \\
\hline $\mathrm{T}$. & 5 & 26 & 10 & 26,6 & 151 & 60,4 & 6 & $\begin{array}{c}0,7 \\
\text { 9. u. } 10 . \text { St. }\end{array}$ \\
\hline
\end{tabular}


Die Zahlen der letzten Spalte zeigen uns die Intensität des Verdauungsprocesses während der zwei Stunden, um die jedes einzelne Experiment das unmittelbar vorangehende an Dauer übertrifft. Ich erhielt dieselben, indem ich jedes Mal von dem mittleren Gewichtsverlust der Eiweisskuben des betreffenden Versuches, den mittleren Gewichtsverlust derselben in dem unmittelbạr vorangehenden subtrahirte und den also erhaltenen Werth dann durch 2 (der Zabl der Stunden entsprechend) dividirte. Diese Zahlen sind es, die uns in deutlichen Zügen den zeitlichen Verlauf des gesammten Processes vorführen. Während der Gewichtsverlust in den zwei ersten Stunden $31 \%$, also $15,8 \%$ in der Stunde beträgt, ist derselbe bereits in der

Fig 1. Verlauf der proteolitischen Wirkung des Pankreassaftes nach dem Tode.

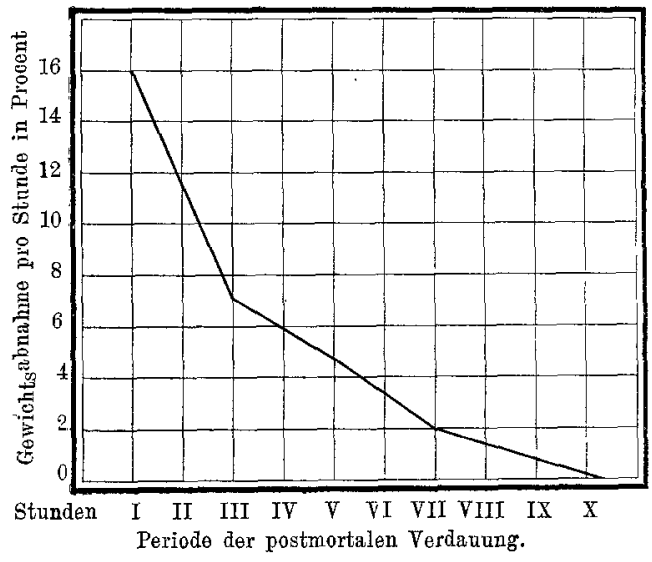

dritten und vierten auf $7 \%$ reducirt, und in der siebenten und achten sogar schon auf 4,7\%. Ueber diese Zeit hinaus erstreckt sich die proteolitische Wirkung kaum; in der 7. und 8. Stunde beträgt der Gewichtsverlust der Eiweisskuben thatsächlich nur $2 \%$, und während der 9. und 10. Stunde ist er auf $0,7 \%$ beschränkt.

Freilich können diese Zahlen nur auf eine annähernde Genauigkeit Anspruch machen, doch erscheint immerhin die Annahme berechtigt, dass mit der 8 . Stunde jegliches Verdauungsvermögen im Darme so gut wie erlosehen ist. Wenn wir bedenken, dass diese Versucbe in einer Jahreszeit angestellt wurden, wo die äussere Temperatur eine ziemlich hohe war, dürfen wir wohl annehmen, dass unter gleichen Bedingungen die Dauer der postmortalen Verdauung im Duodenum derjenigen im Magen beinahe gleichkommt. 
Wollten wir diese Verhältnisse durch eine Curve darstellen (Fig. 1), so würde der erste Theil derselben, entsprechend den ersten 3-4 Standen postmortaler Verdauung, steil abfallen, um dann allmählich einen minder steilen Verlauf anzunehmen. Ein Vergleich mit der Curve, die dieselben Verhältnisse bei der postmortalen Verdauung im Magen ausdrückt (Fig. 2), würde uns belehren, dass, obgleich beide Curven fast gleichzeitig auf Null herabsinken, ihr Verlauf nichtsdestoweniger ein wesentlich verschiedener, ich möchte fast sagen entgegengesetzter ist, denn während die Curve des Zwölffingerdarmes erst schnell und dann langsamer abfällt, verläuft die Curve des Magens während der ersten 3 Stunden fast horizontal, um dann ganz plötzlich und steil auf Null herabzusinken.

Fig, 2. Verlauf der proteolitischen Wirkung des Magensaftes nach dem Tode.

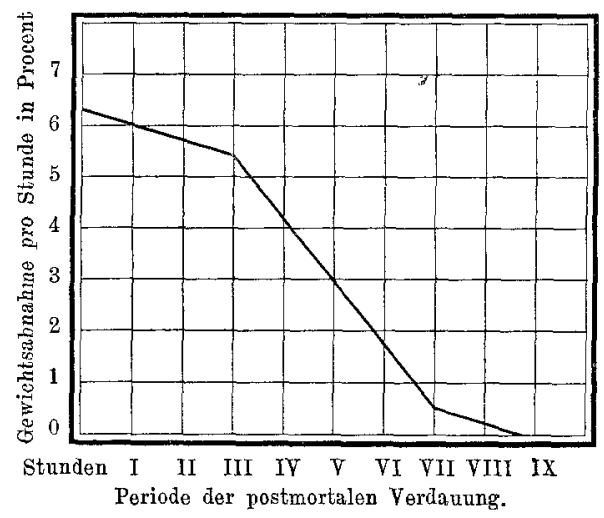

Diese Ergebnisse bieten uns wohl ein dentliches Bild der Art und Weise, wie die Eiweissverdauung im Leichenduodenum verläuft; um jedoch die einzelnen Momente näher zu bestimmen, welche das Verdauungshild in seinen Einzelheiten bedingen, hielt ich es für geboten, neben dem Einfluss der Temperatur auch denjenigen der Anhäufung der Eiweissverdauungsproducte im Darme näher zu untersuchen. Zu diesem Zwecke habe ich eine weitere Versuchsreihe an Hunden unternommen, deren Veröffentlichung ich mir vorläufig noch vorbehalte. Bei diesen Versuchen blieb der Abstand zwischen der in Bezug auf Menge und Qualität identischen Mahlzeit und dem Tode constant, während die Einführung der Eiweisskuben in den Duodenum in immer grösseren Zwischenräumen nach demselben erfolgte.

Um zu crmitteln, in welchem Maasse sich der Einfluss der äusseren Temperatur auf die Intensität der Eiweissverdanung im 
Duodenum der Leiche fühlbar machte, wurde eine zweite, im Folgenden kurz mitgetheilte Versuchsreihe angestelit. Da in unserem Falle auch noch die innere Temperatur der Leiche mit im Spiel war, die sich nur langsam mit der äusseren Temperatur ausgleicht, hätte man erwarten dürfen, dass die Ergebnisse dieser Versuche von den der in vitro vorgenommenen Experimente ganz wesentlich abweichen würden. Es variirte also bei diesen Versuchen, 3 an der Zahl, bloss die äussere Temperatur, während der Zeitraum zwischen Mahlzeit und Tod und die Dauer des Verbleibens der Eiweisskuben im Darm (8 Stuuden) constant blieben. Beim ersten betrug dieselbe 10-12 beim zweiten $16-18^{\circ}$, beim dritten Versuch endlich $32^{\circ} \mathrm{C}$; bei letzterem wurde der Hund in eine auf diese Temperatur erhitzte Wärmekammer gebracht.

\section{Versuch.}

Hund U. Gewicht 51/2. kg. 14. März 1901. Aussere Temperatur $10-12^{\circ} \mathrm{C}$. 8b Morgens: Das Thier bekommt etwas Fleisch. 9h $30^{\prime}$ : Laparotomie; 10 Kuben geronnenes Eiweiss werden auf dem üblichen Wege in den Zwölffingerdarm eingeführt. Auch im Uebrigen verfährt man ganz wie in den vorigen Versuchen. Abends 5 h $30^{\prime}$, alsu 8 Stunden nach dem Tod: Obduction. Die Würfel erscheinen zusammengeschrumpft, gelb verfärbt; der maximale Verlust beträgt 153 , der geringste dagegen $126 \mathrm{mg}$. Die mittlere Gewichtsabnahme ist gleich $137 \mathrm{mg}$, d. h. $55 \%$ und pro Stunde $6,9 \%$.

\section{Versuch.}

Hund V. Geschlecht weiblich. Gewicht $5 \mathrm{~kg}$. 11. Mai 1901. Aeussere Temperatur $16-18^{\circ} \mathrm{C}$.

Um $7 \mathrm{~h} 30^{\prime}$ Morgens wird dem Thier etwas Fleisch verabreicht. $9 \mathrm{~h}$ : Einführung wie oben von 10 Kuben geronnenes Eiweiss in den Zwölffingerdarm; unmittelbar darauf wird das Thier vermittelst Bulbärstiches getödtet. 8 Stunden nach dem Tode: Obduction. Rectaltemperatur $22,5^{\circ} \mathrm{C}$. Die grösste beobachtete Gewichtsabnahme stellt sich auf $162 \mathrm{mg}$, die geringste auf 130 ; mittlere Abnahme der 10 Würfel: $141 \mathrm{mg}=55 \%$, pro Stunde $7,1 \%$.

\section{Versuch.}

Hund Z. Gewicht 51/2 kg. 19. März 1901.

8h: Darreichung von etwas Fleisch. Um 9 h $30^{\prime}$ wird das Thier, nachdem die Einführung von 10 Eiweisskuben auf übliche Weise stattgefunden hat, getödtet und in einen Thermostat gebracht, wo die Leiche 8 Stunden bei einer Temperatur von $32^{\circ} \mathrm{G}$. verbleibt. $5^{\mathrm{h}} 30^{\prime}$ Nachm.: Obduction. Rectaltemperatur $33^{\circ} \mathrm{C}$.

Gewichtsverlust der Würfel : maximaler $160 \mathrm{mg}$, minimaler $133 \mathrm{mg}$. Mittlerer Verlust: $146 \mathrm{mg}=58,5 \%$, pro Stunde $7,4 \%$. 
Fügen wir zu diesen 3 Versuchen noch den 4 . ans der ersten Versuchsreihe hinzu (s. S. 531), der sich unter ähnlichen Bedingungen, aber bei einer äusseren Temperatur von $25^{\circ} \mathrm{C}$. abspielte, wird uns folgende Tabelle über die hier waltenden Verbältnisse belehren.

Tabelle II.

\begin{tabular}{|c|c|c|c|c|c|c|c|c|}
\hline \multirow[t]{2}{*}{ Hund } & \multirow{2}{*}{$\begin{array}{c}\mathrm{Ge}- \\
\text { wicht } \\
\mathrm{kg}\end{array}$} & \multirow{2}{*}{$\begin{array}{c}\text { Tem- } \\
\text { peratur } \\
0 \mathrm{C}\end{array}$} & \multirow{2}{*}{$\begin{array}{c}\text { Zwischen } \\
\text { Fleisch- } \\
\text { mahlzeit u. } \\
\text { Tödtung } \\
\text { verflossene } \\
\text { Zeit } \\
\text { Stunden }\end{array}$} & \multirow{2}{*}{$\begin{array}{l}\text { Verbleib } \\
\text { d. Kuben } \\
\text { im Duo- } \\
\text { denum } \\
\text { Stunden }\end{array}$} & \multirow{2}{*}{$\begin{array}{l}\text { Rectal- } \\
\text { tempera- } \\
\text { tur z. Zeit } \\
\text { d. Obduc- } \\
\text { tion }{ }^{0} \mathrm{C} \text {. }\end{array}$} & \multicolumn{2}{|c|}{$\begin{array}{l}\text { Totale mittlere } \\
\text { Gewichtsabnahme } \\
\text { per } 250 \mathrm{mg}\end{array}$} & \multirow{2}{*}{$\begin{array}{c}\text { Mittlere } \\
\text { Gewichts- } \\
\text { abnahme } \\
\text { p. Stunde } \\
\% \%\end{array}$} \\
\hline & & & & & & $\mathrm{mg}$ & $\%$ & \\
\hline U. & $5^{1 / 2}$ & $10-12$ & $1^{1 / 2}$ & 8 & 21,4 & 137 & 55 & 6,9 \\
\hline V. & 5 & $16-18$ & $11 / 2$ & 8 & 22,5 & 141 & 56,5 & 7,1 \\
\hline S. & 6 & 25 & $11 / 2$ & 8 & 26,2 & 148 & 59 & 7,5 \\
\hline Z. & $5^{1 / 2}$ & $\begin{array}{c}32 \\
\text { Thernost. }\end{array}$ & $1^{1 / 2}$ & 8 & 33 & 146 & 58,5 & 7,4 \\
\hline
\end{tabular}

Man ersieht aus dieser Tabelle, dass der Einfluss der äusseren Temperatur auf den Verlauf der postmortalen Verdauung im Duodenum, im Gegensatz zu meiner früher, auf Grund der trügerischen Ergebnisse einiger Präliminarversuche geäusserten Meinung, ein sehr geringer sein muss. Unter gleichen Bedingungen wäre der Einfluss der äusseren Temperatur auf die Verdaung im Magen ein viel tiefgreifenderer gewesen.

Ich glaube den Grund dafür darin suchen zu dürfen, dass die ausgiebigste Verdauung des Eiweisses im Duodenum während der 3 ersten Stunden post mortem stattfindet, wie dies aus der ersten Tafel deutlich ersichtlich ist, während im Magen das Gegentheil geschieht. Aber gerade während dieser Zeit wird die äussere Temperatur, wie niedrig sie auch sein mag, kaum im Stande sein, die innere Temperatur der Leiche dermaassen herabzusetzen, dass eine Schwächung der proteolitischen Wirkung des Pankreassaftes zu Stande kommen kann, denn nach 3 Stunden ist die Temperatur im Rectum noch nicht viel unter $30^{\circ}$ gesunken. Der Einfluss der äusseren Temperatur erstreckt sich erst auf eine spätere Periode, in der bereits die proteolitische Wirkung des Duodenuminhaltes fast vollständig erschöpft ist, so dass die dadurch bewirkte Schwächung in derselben kaum mehr fühlbar ist.

In einer dritten Versuchsreihe liess ich, bei ungefähr constanter äusserer Temperatur und gleich langem Verbleiben der Eiweisskuben 
im Darme (6 Stunden), den Zeitraum zwischen der Mahlzeit und der Einführung der Kuben in den Duodenum (und gleichzeitiger Tödtung der Thiere) variiren. Bei den ersten 8 Versuchen wurden die Thiere in immer grösseren Zeiträumen von $1 / 2,1,1^{1 / 2}, 2,2^{1 / 2}$, $3,3^{1 / 2}, 4$ Stunden nach der Mahlzeit getödtet, in den 4 letzten erst nach $5,6,7$ und 8 Stunden.

In Folgenden gebe eine kurze Beschreibung dieser Versuche:

\section{Versuch.}

Hund $\alpha$. Gewicht ca. 5 kg. 16. December 1900. Aeussere Temperatur $10-13^{0} \mathrm{C}$.

Um $10^{\mathrm{h}}$ Morgens erhält das Thier etwas gesottenes Fleisch. Um 10h $30^{\prime}$, d. h. nach einer halben Stunde vitaler Verdauung, Laparotomie und Einführung von 8 Eiweisskuben in das Duodenum. Unmittelbar darauf wird das

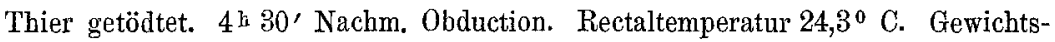
verlust der Eiweisskuben (ursprüngliches Gewicht $250 \mathrm{mg}$ ) nach 6 Stunden postmortaler Verdauung 86, 88,99, 92,97, 100, 82, 95; mittlerer Verlust 92,5= $37 \%$, pro Stunde $6,2 \%$.

\section{Versuch.}

Hund $\beta$. Gewicht $5^{1 / 2} \mathrm{~kg}$. 2. Februar 1901. Aeussere Temperatur $12^{\circ} \mathrm{C}$. Das Thier frisst Morgens $10^{\mathrm{h}}$ etwas gesottenes Fleisch. Um $11^{\mathrm{h}}$ (1 Stunde nach der Mahlzeit): Laparotomie, Eröffnung des Magens und Einführung von 8 Eiweisskuben in das Duodenum. Die Bauchwand wird zugeklemmt und das Thier sofort getödtet. $5^{\mathrm{h}} \mathrm{Nachm}$. (6 Stunden seitdem die postmortale Verdauung im Gang ist): Obduction. Rectaltemperatur $24^{\circ} \mathrm{C}$. Gewichtsverlust der Eiweisskuben 102, 119, 116, 103, 99, 97, 106, 124 mg. Mittlerer Verlust $108,3=43,3 \%$, pro Stunde $7,2 \%$; im Vergleich zum vorigen Versuch wurde die Reduction der Eiweisskuben während desselben Zeitraums postmortaler Verdauung um $6,3 \%$ grösser.

\section{Versuch.}

Hund $\gamma$. Gewicht ca. $6^{1 / 2} \mathrm{~kg}$. 22. Januar 1901. Aeussere Temperatur $10-12^{\circ} \mathrm{C}$.

$9 \mathrm{~h} 30^{\prime}$ wird das Thier mit etwas gesottenem Fleisch gefüttert. Um $11^{\mathrm{h}}$, $1^{1 / 2}$ Stunde nach der Mahlzeit, werden auf die übliche Weise 8 Kuben geronnenes Fiweiss in den Zwölffingerdarm des Thieres eingeschoben und dasselbe hierauf sofort vermittelst Bulbärstiches getödtet. Die Section erfolgt 6 Stunden nach dem Tode, Nachm. $5^{\mathrm{h}}$. Rectaltemperatur $27^{\circ} \mathrm{C}$. Die Volumenreduction der Kuben ist erheblich. Die Gewichtsabnahme beträgt bei den einzelnen Würfeln: $119,128,197,130,129,118,124,144 \mathrm{mg} ;$ mittlerer Verlust $128=51 \%$, pro Stunde $8,5 \%$; im Vergleich zum vorigen Versuch war die Gewichtsabnahme der Kuben während desselben Zeitraums postmortaler Verdauung (6 Stunden) um $7,8 \%$ grösser. 


\section{2., Fersuch.}

Hund $\delta$. Gewicht $4 \mathrm{~kg}$. 17. December 1900 . Aeussere Temperatur $13-17^{\circ} \mathrm{C}$. $8 \mathrm{~h} 30^{\prime}$ Morgens erhält der Hund etwas gesottenes Fleisch. 2 Stunden nach der Verabreichung desselben, um $10^{\mathrm{h}} 30^{\prime}$, werden durch den Magen 8 Kuben geronnenes Eiweiss von je $250 \mathrm{mg}$ in das Duodenum eingeführt. Die Bauchhöhle wird mit 2 Klemmen verschlossen und das Thier vermittelst Bulbärstiches sofort getödtet. $4^{\text {h }} 30^{\prime}$ Nachm. (6 Stunden post mortem): Obduction. Rectaltemperatur $25^{\circ} \mathrm{C}$. Die erheblich zusammengeschrumpften Kuben erscheinen tief gelb verfärbt. Die Gewichtsabnahme beträgt bei den einzelnen: 153, 166, $147,147,134,158,160,145,137 \mathrm{mg}$; mittlerer Verlust demnach $150 \mathrm{mg}=60 \%$, pro Stunde $10 \%$. Im Vergleich zum vorigen Versuch ist der Gewichtsverlust der Kuben während desselben Zeitraums postmortaler Verdaunng um 8,9\% grösser.

\section{Versuch.}

Hund $\varepsilon$. Geschlecht weiblich. Gewicht ungefähr $5 \frac{1 / 2}{1} \mathrm{~kg}$. 15. Februar 1901. Aeussere Temperatur $11-14^{\circ} \mathrm{C}$.

$7 \mathrm{~h} 30^{\prime}$ Morgens: Fütterung mit etwas gesottenem Fleisch. 21/2 Stunden darauf $(10 \mathrm{~h})$ Laparotomie und Einführung in das Duodenum von 8 Eiweisskuben. Auch im übrigen verfährt man wie oben, und das Thier wird sofort getödtet. $4^{\mathrm{h}}$ Nachm.: Nach 6 Stunden postmortaler Verdauung erfolgt die Section. Rectaltemperatur $24,6^{\circ} \mathrm{C}$. Das ursprüngliche Gewicht von $250 \mathrm{mg}$ ist bei den einzelnen Kuben un 179, 195, 182, 186, 169, 180, 173, $170 \mathrm{mg}$ reducirt. Mittlerer Verlust $178 \mathrm{mg}=71,2 \%$, pro Stunde $11,8 \%$. Die Verdauung des geronnenen Eiweisses ist um 11,2\% stärker als bei dem Hunde im vorigen Versuch.

\section{Versuch.}

Hund $\zeta$. Ca. $6 \mathrm{~kg}$ schwer. 17. Februar 1901. Aeussere Temperatur $12-14^{\circ} \mathrm{C}$. $7 \mathrm{~h} 30^{\prime}$ Morgens wird dem Thier etwas gesottenes Fleisch dargereicht. 3 Stunden darauf, um $10^{\mathrm{h}} 30^{\prime}$ wird das Thier vermittelst Bulbärstiches getödtet; nachdem ihm zuvor, in der üblichen Weise, 8 Kuben geronnenes Eiweiss $(250 \mathrm{mg})$ in das Duodenum eingeführt worden sind und die Bauchhöhle zugeklemmt wurde. 6 Stunden nach dem Tode, 4 h $30^{\prime}$ Nachm., nachdem die Temperatur im Rectum bereits auf $23,5^{\circ}$ gesunken ist, findet die Section statt. Das Volumen der Kuben, die im Uebrigen das gleiche Aussehen wie im vorigen Versuche zeigen, ist ausserordentlich reducirt. Gewichtsverlust der einzelnen Kuben: 187, 183, 161, 177, 178, 187, 186, $181 \mathrm{mg}$; mittlerer Verlust $180 \mathrm{mg}=$ $72 \%$, pro Stunde $12 \%$. Im Vergleich zum Hunde $\delta$ ist der Gewichtsverlust der Kuben um 0,8\% grösser.

\section{Versuch.}

Hund $\eta$. Ca. $5 \mathrm{~kg}$ schwer. 19. Februar 1901. Aeussere Temperatur $13-15^{\circ} \mathrm{C}$. Um $7 \mathrm{~h}$ frisst das Thier etwas gesottenes Fleisch. Um $10^{\mathrm{h}} 30^{\prime}, 31 / 2$ Stunden nach dessen Verabreichung: Laparotomie und Einführung der Kuben in das Duodenum durch den Magen wie gewöhnlich. $4^{\text {h }} 30^{\prime}$ Nachm.: 6 Stunden post mortem: Obduction. Rectaltemperatur $25^{\circ} \mathrm{C}$. Kuben, im Aussehen: wie oben. Gewichtsverlust (ab $250 \mathrm{mg}$ ) der einzelnen Kuben: 172, 178, 169, 188, 180, 167, 
$170,165 \mathrm{mg}$; mittlerer Verlust $173 \mathrm{mg}=69,2 \%$, pro Stunde $11,5 \%$; während desselben Zeitraums postmortaler Verdaunng war der Gewichtsverlust des geronnenen Eiweisses diesmal im Vergleich zum vorigen Experiment um 2,8\% grösser.

\section{Versuch.}

Hund 9. Gewicht ca. 51/2 kg. 22. Februar 1901. Aeussere Temperatur $10-12^{\circ} \mathrm{C}$

Um $7^{\mathrm{h}} 30^{\prime}$ Morgens bekommt der Hund etwas gesottenes Fleisch zu fressen. 4 Stunden nach der Fütterung werden 8 Kuben Eiweiss in der üblichen Weise in das Duodenum eingeführt. Unmittelbar darauf wird das Thier durch Bulbärstiche getödtet. 6 Stunden nach dem Tode (5h $30^{\prime}$ Nachm.) erfolgt die Section. Starke Volumenreduction und Färbung der Kuben. Die Gewichtsabnahme bei denselben betragen: $170,191,171,175,177,184,188,176 \mathrm{mg}$; mittlere Abnahme $178=71,2 \%$, pro Stunde $11,8 \%$. Im Vergleich zum vorigen Versuch ist der Gewichtsverlust der Kuben während derselben Verdaungsperiode post mortem $\mathrm{nm} 2 \%$ grösser.

\section{Versuch.}

Hund ¿. Geschlecht weiblich. Gewicht ungefähr 6 kg. 25. Februar 1901. Aeussere Temperatur $10-13^{\circ} \mathrm{C}$.

$7 \mathrm{~h}$ Morgens lasse ich dem Thier etwas gesottenes Fleisch verabreichen: Die Einführung von 8 Kuben geronnenes Eiweiss in das Duodenum durch den Magen erfolgt auf übliche Weise 5 Stunden nach der Mahlzeit. Unmittelbar darauf wird das Thier vermittelst des Bulbärstiches getödtet. 6 Stunden post mortem ( 6 h Abends): Obduction. Rectaltemperatur $23,1^{\circ}$ C. Die Kuben sind immer noch durch Galle verfärbt, ihr Volumen jedoch erscheint in geringerem Maasse reducirt als im vorigen Versuch. Der Gewichtsverlust beträgt bei den einzelnen Kuben: 157, 167, 152, 148, 144, 160, 147, $151 \mathrm{mg}$; mittlerer Verlust $152 \mathrm{mg}=60,8 \%$, pro Stunde $10,1 \%$. Der mittlere Gewichtsverlust des geronnenen Eiweisses war also im Vergleich zum vorigen Versuch um 10,4\% geringer.

\section{Versuch.}

Hund $x$. Ca. $5 \mathrm{~kg}$ schwer. 5. März 1901. Aeussere Temperatur $13-15^{\circ} \mathrm{C}$.

Um $7^{\mathrm{h}}$ Morgens frisst das Thier etwas gesottenes Fleisch. Nachm. $1^{\mathrm{h}}$ wird es getödtet, unmittelbar nachdem auf die übliche Weise die Einführung von 8 Kuben geronnenen Eiweisses in den Zwölffingerdarm erfolgt ist.

Abends $7^{\text {h }}$ (sechs Stunden post mortem): Section. Rectaltemperatur $23,9^{\circ} \mathrm{C}$. Volumenreduction der Kuben noch geringer, Färbung heller. Gewichtsverlust bei den einzelnen Kuben: $138,131,125,136,121$, 116, 121, $130 \mathrm{mg}$; mittlerer Gewichtsverlust $127,5=51 \%$, pro Stunde $8,5 \%$. Die Verdauung der Kuben ist demnach um $9,8 \%$ geringer als im vorigen Versuch.

\section{Versuch.}

Hund $\lambda$. Gewicht ca. $4 \frac{1 / 2}{\mathrm{~kg}}$. 27. Màiz 1901. Aenssere Temperatur $12-15^{\circ} \mathrm{C}$. $9 \mathrm{~h}$ Morgens frisst der Hund etwas gesottenes Fleisch. $4 \mathrm{~h}$ Nachm. (7 Stunden nach der Mahlzeit), erfolgt die Laparotomie und Einführung in das Duodenum 
der 8 Eiweisskuben. Hierauf wird das Thier sofort vermittelst Bulbärstiches getödtet. $10^{\mathrm{h}}$ Abends (nach 6 Stunden postmortaler Verdauung): Obduction. Rectaltemperatur $26^{\circ}$ C. Die Volumenreduction der Kuben ist eine erheblich geringere als bei den vorigen Experimenten. Die Gewichtsabnahme der einzelnen Kuben beträgt bloss 108, 101, 112, 99, 104, 106, 93, $117 \mathrm{mg}$; mittlere Abnahme $105 \mathrm{mg}=42 \%$, pro Stunde $7 \%$. Im Vergleich zum vorigen Versuch ist die Gewichtsabnahme der Eiweisswürfel während desselben Zeitraums postmortaler Verdauung um $9 \%$ geringer.

\section{Versuch.}

Hund $\mu$. Gewicht ca. 5 kg. 29. März 1901. Aeussere Temperatur $13-15^{\circ} \mathrm{C}$.

$8 \mathrm{~h}$ Morgens bekommt das Thier etwas gesottenes Fleisch. 8 Stunden darauf (um $4^{\mathrm{h}} \mathrm{Nachm}$ ) werden auf dem üblichen Wege 8 Eiweisskuben $(250 \mathrm{mg}$ ) in den Zwölffingerdarm eingeführt und der Hund unmittelbar darauf getödtet. 10 h Abends ( 6 Stunden nach dem Tode) erfolgt die Section. Die Temperatur im Rectum beträgt $25,2^{\circ} \mathrm{C}$. Die Kuben erscheinen nur in geringem Maasse reducirt, auch ist die Färbung eine hellere als bei den vorigen Versuchen. Der Gewichtsverlust der einzelnen Kuben beträgt: $78,81,89$, 77, 85, 82, 79, $95 \mathrm{mg}$; mittlerer Verlust $83,3=33,3 \%$, pro Stunde $5,6 \%$. Im Vergleich zum vorigen Versuch ist die mittlere Abnahme der Kuben in Folge der Eiweissverdauung um 8,7\% geringer.

Auf diese 12 Versuche liess ich einen dreizehnten folgen, bei welchem der Hund kein Fleisch zu fressen bekommen hatte, vielmebr seit 24 Stunden nüchtern war.

Dieser Versuch schien mir besonderes Interesse zu gewähren; es ist bekannt, dass die Meinungen der verschiedenen Forscher ( $\mathrm{C}$ or visart, Schiff, Herzen, Carvallo et Pachon, Dastre) betreffs des Inhaltes des Pankreas der nüchternen Thiere, an Fermenten und der proteolitischen Wirkung des unter diesen Bedingungen abgesonderten Pankreassaftes, nicht übereinstimmen; was letztere betrifft, wird sie von dem Einen entschlossen in Abrede gestellt, während Andere wieder nachgewiesen haben, dass der in Folge Erregung des Vagums abgesonderte Saft der Pankreasfistel auch bei nüchternen Thieren eine proteolitische Wirkung zu entfalten im Stande ist (Wett), und Pachon et Gachet das in das Duodenum lebender, nüchterner Thiere eingeführte Eiweiss nach 5-6 Stunden vollkommen zersetzt fanden. Der Versuchsverlauf war folgender:

Hund $\alpha^{\prime}$. Geschlecht weiblich. Gewicht 51/2 kg. 15. Mai 1901. Aeussere Temperatur $16-18^{\circ} \mathrm{C}$.

$10 \mathrm{~h}$ Morgens. Der Hund hat in den letzten 24 Stunden nichts zu fressen bekommen. Laparotomie und Einführung von 8 Kuben Eiweiss in das Duodenum durch die untere Magenöffnung. Nachdem die Bauchhöhle zugeklemmt, wird das Thier vermittelst Bulbärstiches getödtet. $4^{\mathrm{h}}$ Nachm.: Obduction. Temperatur im Rectum 24,70 C. Gewichtsverlust der Kuben 51, 53, 85, 62, 58, 79, 64,60 ; mittlerer Verlust $64=25,6 \%$, pro Stunde $4,3 \%$. 


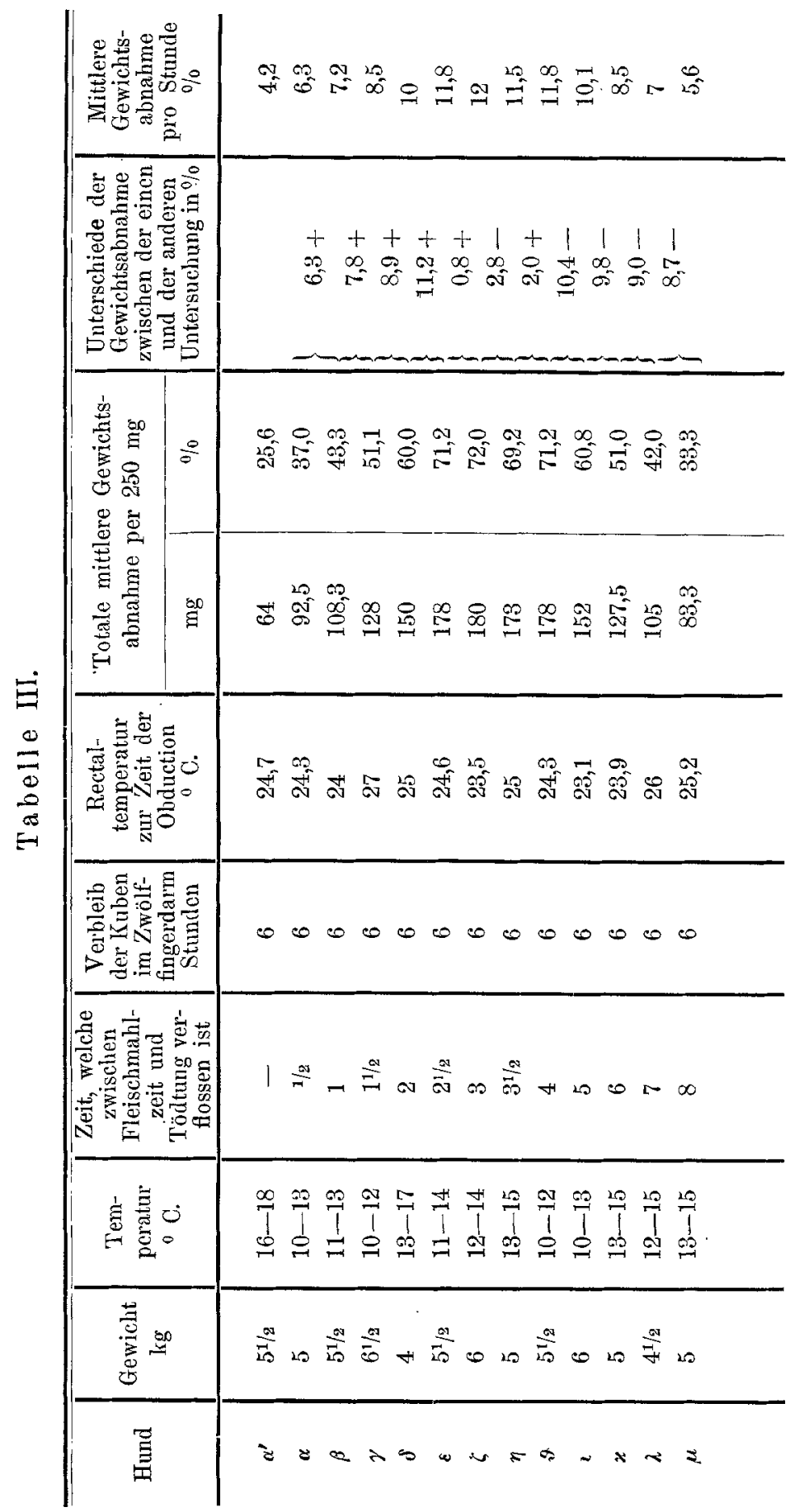


Unerwarteter Weise lautet das Ergebniss auf einen Verbrauch von Eiweiss, aber in beschränkterem Maasse als bei verdauenden Thieren. Die Bildung eines Duodenalsaftes mit proteolitischer Wirkung kann verschiedenartig gedeutet werden; da die Annahme eines Fortdauerns der Pankreasfunction als unberechtigt erscheinen kann, sind wir auf die verschiedenen Momente angewiesen, die auf das Thier vor und während des Todes einwirken können, so z. B. die unvermeidliche psychische Erregung, wenn das nüchterne Thier von demselben Manne aus dem Stall fortgeschleppt wird, der ihm gewöhnlich das Futter darreicht, oder den unmittelbaren mechanischen Reiz durch die Einführung der Kuben (was jedoch nach den Untersuchungen von Paulow's Schülern unwahrscheinlich erscheint) oder endlich die Erregung des Bulbus bei der Tödtung (wie bekannt, fanden Heiden$\mathrm{h}$ a in und Landa $\mathrm{u}$, dass eine elektrische Erregung des Bulbus auf die $\Lambda$ bsonderung des Pankreassaftes befördernd wirkt).

Die Ergebnisse der ganzen Versuchsreihe sind in der Tabelle (S. 540) zusammengestellt. In der vierten Spalte ist die jedesmalige Dauer der vitalen Verdauung angegeben, worauf das Thier getödtet und die Eiweisskuben in das Duodenum eingeführt wurden, in der fünften die Dauer der postmortalen Verdaung, die in allen Fällen von 6 Stunden war. In der vorletzten Spalte ist der positive oder negative Unterschied des Eiweissverbrauches (in Procenten) zwischen dem betreffenden Versuche und dem unmittelbar vorangehenden angegeben.

Wie daraus ersichtlich, entfaltet somit der Duodenuminhalt das Maximum seiner verdauenden Thätigkeit bei solchen Thieren, die $2^{1 / 2}$ bis 4 Stunden nach der Fleischverfütterung getödtet werden. Zu dieser Zeit beträgt die Verdauung selbst $72 \%$ der gesammten Eiweissmenge.

Der Verlauf der proteolitischen Wirkung des Duodenuminhalts im Laufe der Verdauung ist klar ersichtlich aus folgender Curve, die unter Zugrundelegung der in voriger Tafel enthaltenen Angaben gezeichnet wurde. Anfangs steigt dieselbe steil an, so dass ihr ansteigender Schenkel von einer geraden, beinahe verticalen Linie dargestellt wird. Ihren Höhepunkt erreicht sie nach etwa $2^{1 / 2}$ Stunden und verbleibt während ca. 11/2 Stunden auf demselben, um dann wiederum rasch, jedoch nicht so schroff wie im ansteigenden Schenkel, abzufallen. Die Schwankungen, die man am Höhepunkt der Curve beobachtet, sind sicherlich durch eines der mannigfachen Momente 
bedingt, die den Versuchsverlauf beeinflussen konnten, trotzdem möglichst dafür Sorge getragen wurde, die grösste Beständigkeit der Versuchsbedingungen zu erzielen.

Es dürfte sich lohnen, diese Ergebnisse mit den gleich bezüglichen Paulow's und seiner Schüler zu vergleichen. Fassen wir

Fig. 3. Variation der proteolitischen Wirkung des Duodenalsaftes in verschiedenen Perioden der Verdaung.

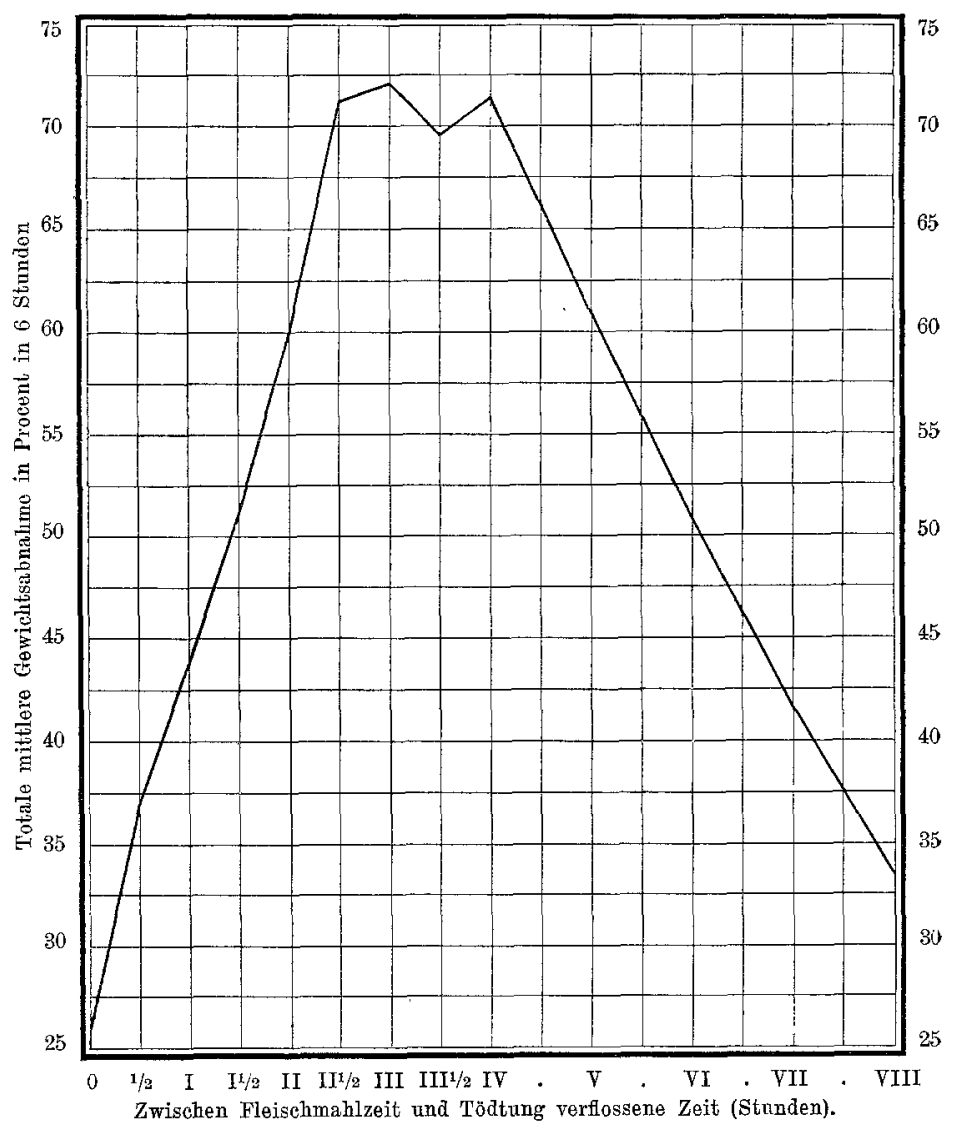

zuerst die Bedingungen in's Auge, unter welchen sich diese grundlegenden und geistvollen Versuche vollzogen, es wurde der Saft von Thieren untersucht, die Träger einer dauernden, nach Paulow's Methode erzeugten Pankreasfistel waren. Unter solchen Umständen können die Thiere gewiss nicht als normal angesehen werden, da die Pankreasfunction seit etlichen Tagen fehlte; zudem verlief auch die Verdauung während der Versuche nicht wie im normalen Thiere, 
weil sich der Pankreassaft nicht in das Duodenum ergoss und die Beschaffenheit und Reaction des Duodenuminhalts somit eine von der normalen verschiedene war und in Folge dessen auch der von demselben ausgeübte, die Pankreasthätigkeit befördernde Reiz ausblieb.

Auch nach dem Verfahren von Foderà wären diese Missstände nicht gehoben worden. Nach dieser Methode ergiesst sich der Pankreassaft durch ein T-förmiges Rohr für gewöhnlich in das Duodenum, kann aber beliebig auch nach aussen geführt werden; es fällt dadurch leicht, sich einen klaren Begriff von den qualitativen Veränderungen desselben zu machen, die quantitativen jedoch entziehen sich einer klaren Würdigung, da die Menge des nach aussen fliessenden Secrets in keinerlei bestimmbarer Beziehung zu derjenigen steht, die sich weiter in das Duodenum ergiesst, zudem wird bei solchen Versuchen ein nicht unwesentlicher Theil des Pankreassaftes der Verdauungsfunction entzogen. Nach unserer Methode functionirt der Darm selbst als Behälter und Wärmekammer bei der Reaction, und es wird dadurch ermöglicht, die Wirkung der proteolitischen Fermente direct in demselben und somit in den denkbar normalsten Verhältnissen zu untersuchen. Freilich hat man es dabei nicht mit der Wirkung des Pankreassaftes allein zu tbun, sondern mit derjenigen des gesammten Darminhalts, doch hängt offenbar die Verdauung des geronnenen Eiweisses ausschliesslich von der Wirkung der proteolitischen Fermente ab. Da den von mir erhaltenen Zahlen offenbar kein absoluter, sondern bloss ein relativer Werth beizumessen ist, und in allen Versuchen die Kuben gleich lang im Darme verblieben, wird ihre Vergleichbarkeit keineswegs dadurch beeinträchtigt, dass während der sechs Stunden der postmortalen Verdauung die sich anhäufenden Verdauungsproducte des Eiweisses nothwendig einen hemmenden Einfluss auf die weitere Verdauung des Eiweisses selbst ausüben mussten.

Aus den von Walther zusammengestellten Tabellen lässt sich deutlich ersehen, dass die Intensität der proteolitischen Wirkung des von einer Fistel herruhrenden Pankreassaftes anfangs (von der ersten bis zur dritten Stunde nach der Verabreichung des Fleisches) geringer wird und hierauf zunimmt. Dagegen nimmt die Menge des abgesonderten Pankreassaftes während der ersten Stunde rasch zu, erreicht nach zwei Stunden ihr Maximum und fällt dann wieder schnell $a b$, so dass nach fünf Stunden bereits kein Saft mehr fliesst. 
Letztere Thatsache lässt sich leicht mit den Ergebnissen meiner Versuche in Einklang bringen. Nach zwei Stunden erreicht der Darm seinen maximalen Inhalt an Pankreassaft, und es ist leicht begreiflich, dass sich der schwächende Einfluss der verminderten Saftabsonderung erst lange Zeit, nachdem diese Verminderung wirklich eingetreten ist, geltend machen muss. Daher ist es auch natürlich, dass meine Curve im Grossen und Ganzen derjenigen der secretorischen Thätigkeit ähnlich verlaufe, ihren Höhepunkt jedoch erst.später erreiche, sich aber auf demselben entsprechend länger behaupte.

Um hiergegen meine Ergebnisse mit Wa th er's Bestimmungen der proteolitischen Wirkung des Saftes der Pankreasfistel in Einklang zu bringen, muss man annehmen, dass unter unseren Versuchsbedingungen die Eiweissverdauung eher als von der Concentration der Fermente im Duodenuminhalte von der absoluten Menge derselben darin abhänge, und diese Annahme erscheint um so mehr berechtigt, wenn man den abschwächenden Einfluss der Verdauungsproducte auf die Wirksamkeit des Enzyms berücksichtigt; dass der Gesammtinhalt an Fermenten nicht mit der Concentration des Pankreassaftes Schritt hält, ist um so leichter verständlich, als die Zunahme der Secretmenge unverhältnissmässig grösser ist als die gleichzeitige Abnahme ihres proteolitischen Vermögens, so dass zur Zeit der lebhaftesten secretorischen Thätigkeit und nachher die Ver. dünnung des Duodenalinhalts wohl eine grössere, dafür aber sein absoluter Reichtbum an Ferment ein viel beträchtlicherer ist.

Aus meinen Versuchen ergibt sich somit ein synthetischeres und getreueres Bild der Verdauungsvorgänge im Duodenum, als wenn sich unser Studium bloss auf den aus einer Magenfistel erhaltenen Pankreassaft beschränkt hätte, doch dürfen wir letzterer Methode ihre bedeutenden Vortheile nach einer anderen Seite hin nicht absprechen.

Meine Ergebnisse könnte man somit dahin zusammenfassen:

Führt man in das Duodenum eines Hundes, bei dem der Verdauungsprocess in vollem Gange ist, eine gewisse Menge geronnenes Eiweiss ein, indem man das Thier gleichzeitig tödtet, so wird dieses Eiweiss in hohem Maasse verdaut. In den verschiedenen Zeiträumen nach dem Tode ist diese Verdauung nicht etwa eine gleichmässige, vielmehr fällt die höchste Intensität derselben in die ersten Stunden post mortem, um dann rasch abzunehmen und nach ungefähr 
neun Stunden gänzlich zu schwinden. Diese Abnahme ist nicht bloss abhängig von der Erkaltung der Leiche, sondern auch von anderen, noch nicht genauer ermittelten Momenten.

Die äussere Temperatur übt auf die Intensität dieser postmortalen Verdauung im Duodenum bloss einen beschränkten Einfluss aus.

Je nachdem zur Zeit der Einführung des Eiweisses in den Darm die Verdaung der vorangegangenen Fleischmahlzeit mehr oder weniger vorgeschritten ist, schwankt die Intensität der Verdauungsprocesse im Duodenum der Thierleiche bei gleicher äusserer Temperatur und gleich langem Verbleiben des Eiweisses im Darme innerhalb ziemlich weiter Grenzen; sie steigt rasch bis auf ein Maximum, das erreicht wird, wenn der Tod in einem Abstande von ca. $2^{1 / 2}$ Stunden auf die Mablzeit folgt. Sind zwischen Mahlzeit und Tod mehr als vier Stunden verflossen, bemerkt man eine allmähliche Abschwächung der Verdaungsprocesse.

Die jeweilige proteolitische Wirkung des Duodenuminhalts während der Verdauung hängt nicht von der Concentration der Fermente in demselben, wie sie aus dem Verlaufe dieser Wirkung im Safte der Pankreasfistel erhellt, ab, sondern vielmehr von der absoluten Menge activer Fermente, die zur Zeit im Darme vorhanden ist.

Auch der Duodenuminhalt eines nüchtern getödteten Thieres vermag auf das gleich beim Tode eingeführte geronnene Eiweiss eine verdauende Wirkung anszuüben.

\section{Literatur.}

Carvallo et Pachon, Expériences sur le pouvoir digestif du pancréas dans l'état de jeune chez les animaux normaux et dératés. Arch. de Physiol. t. 5.1893.

L. Corvisart, Infuence de la digestion gastrique sur l'activité fonctionelle du pancréas. Union médicale 1861.

A. Dastre, Contribution a l'étude des ferments du pancréas. Arch. de Physiol. t. 5. 1893.

C. Ferrai, Ueber postmortale Verdauung. Vierteljahrsschrift f. gerichtl. Medicin u. öff. Sanitätsw. 3. Folge Bd. 21 S. 2.

Foderà, Moleschott's Untersuchungen zur Naturlehre der Menschen und der Thiere Bd. 16. 1896. 
546 C. Ferrai: Zur Kenntniss der Duodenalverdauung des Eiweisses etc.

Landau, Zar Physiologie der Bauchspeichel-Absonderung. Breslau 1873.

A. Herzen, Ueber den Einfluss der Milz auf die Bildung des Trypsins. Pflüger's Archiv Bd. 30. 1883.

J. Jablonski, Comment se comporte la glande pancréatique sous l'infinence du régime pano-lacté. Arch. des Sciences biologiques de St. Pétersbourg t. 4. 1895.

S. Mett, Zur Innervation der Bauchspeicheldrüse. Arch. f. Anatomie u. Physiologie 1894.

V. Pachon et J. Gachet, Du pouvoir digestif du duodenum vis-à-vis de l'ovoalbumine. Compt. rend. de la Soc. de Biologie Sér. 10 t. 4. 1897.

J. P. Paulow, Die Arbeit der Verdauangsdrüsen. Bergmann, Wiesbaden 1898.

J. P. Paulow, Die Methode der Anlegung einer Pankreasfistel. Verhandlungen der St. Petersburger Naturforschergesellschaft Bd. 11. 1879. HofmannSchwalbe, Jahresbericht f. 1880.

M. Schiff, Pankreasverdaung. Pflüger's Archiv 1870.

A. Ssamoilow, Determination du pouvoir fermentatif des liquides contenants de la pepsine par le procédé de M. Mett. Arch. d. Scienc. biologiques de St. Pétersbourg t. 2. 1893.

A. Walther, Excitabilité secretoire spécifique de la mucquese du tube digestif. $5^{\text {me }}$ mémoire. Sécretion pancréatique. Arch. d. Scienc. biologiques de St. Pétersbourg t. 7. 1898.

W. Was siliew, De l'influence des diverses espèces d'aliments sur le fonctionnement de la glande pancréatique. Arch. des Sciences biologiques de St. Pétersbourg t. 2. 1898. 